Magdolna Gedeon

Die Rechtslage des Grundbesitzes im Spiegel

der Lehre von der Heiligen Krone
Journal of Agricultural and

Environmental Law

$29 / 2020$

\title{
Magdolna Gedeon* \\ Die Rechtslage des Grundbesitzes im Spiegel der Lehre von der Heiligen Krone
}

Abstract

In relation to the Doctrine of the Holy Crown there are two opposing views. In one of the opinions the state power and the lands belonged to the Holy Crown, and for this reason the decisions about the lands had to be made by the ruler and the orders together. In the other opinion the state power and the lands belonged to the ruler, and for this reason the relation between the king and the person who was donated with a land was a private legal connection. Although the mineral resources originally belonged to the Holy Crown, in the course of time the Habsburg rulers achieved that they could dispose alone, without the consent of the orders, over the mine revenues. In this way, by the 18th century, the relation between the king and the mining contractors became a private legal connection. Comparing the rules of the Bergregal and the rules related to the lands we can conclude that the lands belonged to the Holy Crown, and not to the ruler.

Keywords: Doctrine of the Holy Crown, lands, Bergregal, Habsburgs

Über die Lehre von der Heiligen Krone wird auch heute viel diskutiert. Hat diese in unseren Tagen einen Sinn? Ist diese Lehre eine mythische Theorie, oder ein mittelalterlicher Überrest? Kann die Lehre von der Heiligen Krone auch heute eine Basis für die Rechtskontinuität bilden?

Die Heilige Krone wird auch im ungarischen Grundgesetz erwähnt. ${ }^{1}$ Nach der Meinung von János Zlinszky besteht der heutige Sinn der Lehre von der Heiligen Krone darin, dass sie "das Symbol der im öffentlichen Leben der politischen Nation teilnehmenden Einheit war. Die moderne Formulierung dieser Lehre ist die These der Verfassung, die die Souveränität dem ganzen Volk gibt."2 Diese Souveränitätstheorie spielte eine bedeutende praktische Rolle in der Übung der Hoheitsrechte.

Magdolna Gedeon: Die Rechtslage des Grundbesitzes im Spiegel der Lehre von der Heiligen Krone. Journal of Agricultural and Environmental Low ISSN 1788-6171, 2020 Vol. XV No. 29 pp. 51-65, https://doi.org/10.21029/JAEL.2020.29.51

* dr. jur., PhD, Universitätsdozentin, Universität Miskolc, Staats- und Rechtswissenschaftliche Fakultät, Lehrstuhl für Römisches Recht, e-mail: jogmagdi@uni-miskolc.hu.

${ }^{1}$ Nationales Bekenntnis im Grundgesetz: „Wir halten die Errungenschaften unserer historischen Verfassung und die Heilige Krone in Ehren, die die verfassungsmäßige staatliche Kontinuität Ungarns und die Einheit der Nation verkörpern. “

2 Zlinszky 2013, 227.

https://doi.org/10.21029/JAEL.2020.29.51 


\section{Die „Echart-Polemik“}

In Bezug auf die Lehre von der Heiligen Krone ist die berühmteste Polemik, die zwischen Ferenc Echart und Ákos Timon ${ }^{3}$ entstand. ${ }^{4}$ Echart verfasste auch eine Monographie über die Lehre von der Heiligen Krone, worin er seine These ausführlicher erläuterte. ${ }^{5}$ Nach der Auffassung von Timon knüpfte sich die staatliche Gewalt nicht an die Person des Königs, sondern an die Heiligen Krone: "die staatlichen Hoheitsrechte sind keine königlichen Majestätsrechte mehr, sondern Rechte der Heiligen Krone (iura Sacrae regni Coronae), die prinzipiell der Heiligen Krone zustehen und erst von dieser auf den König übergehen."'6

Das Wesen der übertragenen Gewalt wurde von Werbőczy folgenderweise formuliert: "Als die Ungarn ihn (nämlich Stefan den Heiligen) aus freiem Willen zum König wählten und krönten, wurde das Recht der Erhebung in den Adelsstand, folglich das Recht der Schenkung, wodurch die Adeligen ausgezeichnet und von den NichtAdeligen gesondert werden, vollkommen, zugleich mit der Herrschaft und der Regierung von der Gemeinschaft und Kraft des Willens der Gemeinschaft auf die Heilige Krone dieses Reiches und demzufolge auf unsern Fürsten und König übertragen." ${ }^{\text {"7 }}$

Demzufolge setzte Timon fest: "Das Staatsgebiet ist das Gebiet der Heiligen Krone; die königlichen Einkünfte sind Einkünfte der Heiligen Krone (peculia oder bona Sacrae regni Coronae); jedes freie Besitzrecht stammt von der Heiligen Krone, als der Wurzel (radix omnium possessionum) und fällt daher nach dem Aussterben des Geschlechts an die Heilige Krone heim."

Echart meinte aber, dass Werbőczy “die organische Ansicht der Heiligen Krone nur für den Beweis der These der gleichen adeligen Freiheit verwandte, sonst stand er auf dem Grund der in der alten historischen Entwicklung ausgebildeten Kronenlehre, und der aus den Chroniken übernommenen Volkssouveränität."’

\footnotetext{
3 Ákos Timon (1850-1925) war der Vertreter der historischen Rechtsschule. Er betonte die Originalität der ungarischen Rechtsentwicklung, deren Bedeutung und Wirkung nur mit der englischen Rechtsentwicklung vergleichbar war. Die Ansichten über die ungarische Rechtsentwicklung von Timon wurden auch in seiner Zeit kritisiert (Vgl. Bódi 2015, 31). Timon erörterte seine These über die Heilige Krone in den Werken: Timon 1907, Timon 1920. Die wichtigsten Thesen der Lehre von der Heiligen Krone sind auch in seinem Lehrbuch auffindbar: Timon 1904.

4 Ákos Timon starb im Jahre 1925. Ferenc Echart (1885-1957) disputierte mit Folgern von Timon. Über die Polemik siehe ausführlicher: Törő 2016. Die Programmstudie von Echart: Echart 1931.

${ }^{5}$ Echart 1941. Echart versuchte seine These meistens durch die Interpretation des Wortlauts der Urkunden zu unterstützen.

${ }^{6}$ Timon 1904, 512.

7 Tripartitum, tit. 3. p. I. \6. (Übers. Timon 1904, 513).

8 Timon 1904, 513.

${ }^{9}$ Echart 1941, 210.
} 
Magdolna Gedeon

Die Rechtslage des Grundbesitzes im Spiegel

der Lehre von der Heiligen Krone
Journal of Agricultural and

Environmental Law

$29 / 2020$

János Zlinszky erklärte, dass der ungarische Staat schon auch vor Stefan dem Heiligen durch die geteilte, kontrollierte Gewalt geprägt wurde. In Ungarn entwickelte sich nicht auf das System der Privatgewalten basierten Feudalismus, sondern eine öffentlich-rechtliche Ständeordnung. ${ }^{10}$ Zlinszky schreibt über die geteilte Macht folgenderweise: "Zu der Übung der Staatsoberhauptmacht gehört, - wie das im Gesetzbuch und in den Mahnungen des Stefans des Heilligen befestigt wird ${ }^{11}$ - dass der Rat aus der Übung der Macht nicht ausgeschlossen werden darf, und die Freien in ihrer Freiheit behalten werden sollen, ihre Rechte durch Gesetzgebung und Jurisdiktion nicht entzogen werden dürfen. In diesen Prinzipen - gegenüber derjenigen Lehre des Lehnswesens, dass jedermann sich infolge der persönlichen Beziehung unter die Macht des an der Lehnspyramide stehenden Herrscher unterwerfen soll, und gegenüber der kirchlichen Ideologie, wonach der König durch die Salbung und durch die kirchlichen Auswahl nur dem Gott verantwortlich ist, das weder Unverantwortlichkeit noch gemeinsame Macht bedeutet - vertritt Ungarn die geteilte Macht. Der König, der Rat und das aufgerüstete Volk üben die Macht gemeinsam. Dieser Gedanke wird auch offensichtlich nicht in der späteren Ausführlichkeit - in den Artikeln von Stefan dem Heiligen festgesetzt."12

\section{Die Änderungen der Rechtslage des Grundbesitzes}

Nach der Auffassung, die der Lehre von der Heiligen Krone eine starke öffentlich-rechtliche Bedeutung voraussetzt, gehörte der Bodenbesitz nicht zu der Person des Königs, sondern zur Heiligen Krone. Zwischen dem König und dem Grundbesitzer gab es keine privatrechtliche, sondern eine öffentlich-rechtliche Beziehung. Imre Hajnik erklärte, dass der Grundbesitz in seiner Zeit vor allem volkswirtschaftliche Bedeutung und infolgedessen auch soziale und politische Wirkung hatte. Im mittelalterlichen feudalen Europa hatte der Grundbesitz auch besondere politische Relevanz. Die Ständeordnung und die Regierung wurden nämlich mit dem Grundbesitz verknüpft. Die wichtigeren politischen und gesellschaftlichen Tätigkeiten, wie auch die Kriegsdienstleistung, basierten am Grundbesitz. Hajnik meinte aber, dass die Lehnspyramide mit der Ständeordnung in Ungarn zur Zeit des Stefans des Heiligen nicht gleichgestellt werden darf. Der Grundbesitz zog nach sich in Ungarn nämlich den Unterschied zwischen den vollen und den nicht ganz vollen Freien, aber das führte sich nicht zur Differenz zwischen der Freien. ${ }^{13}$

\footnotetext{
10 Zlinszky 2013, 133-134.

${ }^{11}$ In den Mahnungen des Königs Stefan des Heiligen an seinen Sohn Emerich wird mehrmals die Krone solcherweise erwähnt, als der Träger der Macht. Der König schließt die Mahnungen folgenderweise: "Die königliche Krone wird durch die Gesamtheit der oben Gesagten geschaffen, ohne die niemand auf der Erde regieren und in den Himmel kommen kann" (Érszegi 1987, 61).

12 Zlinszky 2013, 227-228.

13 Hajnik 1867, 29-38.
} 
Magdolna Gedeon

Die Rechtslage des Grundbesitzes im Spiegel

der Lehre von der Heiligen Krone
Journal of Agricultural and

Environmental Law

$29 / 2020$

Ferenc Echart stritt aber die Rückführungsbarkeit der Lehre von der Heiligen Krone bis Stefan den Heiligen. Er meinte, dass die ungarische Verfassungsentwicklung nicht mehr oder weniger durch das öffentliche Recht geprägt wurde, als die übrigen europäischen Verfassungen. Er vermutete deshalb das privatrechtliche Lehnsverhältnis zwischen dem König und dem Grundbesitzer: "Die lehnsrechtliche Auffassung hatte eine große Bedeutung auch in der ungarischen Rechtsentwicklung. Die Entstehung unserer Verfassung wurde unter der Wirkung der lehnsrechtlichen Auffassung, und nicht unter der Ausrichtung eines eigenartigen, ungarischen öffentlich-rechtlichen Geistes vollgezogen. Dadurch wurde auch bewiesen, dass wir uns der Wirkung der allgemeinen mittelalterlichen Kultur auch am diesem Gebiet nicht entzogen."14 Echart setzte fest, dass das Donationssystem diejenige Rechtsauffassung über das Verhältnis zwischen dem Herrscher und den Untertanen erstellte, die durch das Lehnswesen in Westeuropa geschafft wurde. Er meinte, dass die mittelalterlichen Menschen keine öffentlich-rechtliche Gesinnung hatten. Diese Menschen betrachteten den König als den obersten Besitzer, den Besitzer aller Boden, von dem alle Bodenbesitz stammt. ${ }^{15}$

Timon schreibt aber Folgendes: "Das ungarische Volk begeisterte sich nicht für ein Individuum, oder ein Individuen; sondern für Ideen für die Gesamtheit. Die natürliche Folge hiervon war, dass bei den Ungarn das Prinzip der Individualität sich nie über das Staatsprinzip emporschwingen konnte und dass im öffentlichen Leben der Ungarn die privatrechtliche Richtung gegenüber der öffentlich-rechtlichen stets unterliegen musste."16

Nach Echart symbolisierte die Krone die der Person des Königs zustehende Gewalt. Er anerkannte die 'bleiche' Differenzierung der Krone und der Person des Königs in Bezug auf die unveräußerlichen Güter, er meinte aber, dass es aus keinen öffentlich-rechtlichem Aspekt erfolgte, sondern das war die Garantie der Beibehaltung der königlichen ererbten Gewalt. Die heutigen wissenschaftlichen Forschungen sehen aber in der Bedeutung der unveräußerlichen königlichen Güter die Beschränkung der Gewalt durch die Gemeinde. ${ }^{17}$

Diese Auffassung wird auch durch die Lehre von der Heiligen Krone bestärkt, die von Werbőczy in seinem Tripartitum eindeutig festgesetzt wurde. Timon fast die Lehre von Werbőczy folgenderweise zusammen: "Die unmittelbare Beziehung des freien Besitzrechts auf die Heilige Krone bringt eine neue Basis der Gemeinfreiheit, der Teilnahme an den öffentlichen Rechten: den Begriff der Mitgliedschaft der Heiligen Krone hervor. Wer sein Besitzrecht von der Heiligen Krone herleitet und demnach in unmittelbarer Beziehung zu ihr steht, ist ein Glied der Heiligen Krone (membrum Sacrae regni Coronae) und nimmt als solches an der Ausübung der in der Heiligen

\footnotetext{
${ }_{14}$ Echart 1931, 313. Man soll betonen, dass Echart kein Rechtswissenschaftler war, er suchte die Beweise zu seinen Festsetzungen im Sinn der positiven Denkrichtung in den Urkunden. Er studierte römisches Recht nicht, beherrschte deshalb keine kategorische Denkweise hinsichtlich der Rechtsinstitute. Er konnte die Wesensarten des Eigentums und den Unterschied zwischen den öffentlich- und privatrechtlichen Verhältnissen nicht ganz verstanden (vgl. Zétényi 1997, 144).

${ }_{15}^{15}$ Echart 1941, 315.

16 Timon 1904, 74-75.

17 Tóth 2008, 92.
} 
Magdolna Gedeon

Die Rechtslage des Grundbesitzes im Spiegel

der Lehre von der Heiligen Krone
Journal of Agricultural and

Environmental Law

$29 / 2020$

Krone vereinigten öffentlichen Gewalten: der gesetzgebenden, vollstreckenden und richterlichen Gewalt teil. Neben den Adeligen sind noch die begüterten Kirchen und die Städte Glieder der Heiligen Krone, denn auch diese haben ihre Grundbesitze (iure radicali) von der Heiligen Krone empfangen. Sie alle bilden zusammen mit dem König, dessen Haupt die Heilige Kröne heiligt, jenes einheitliche, öffentlich-rechtliche Ganze, jenen lebendigen Organismus, den die mittelalterlichen Quellen als den ganzen Körper der Heiligen Krone (totum sacrae regni Coronae) bezeichnen, und den wir unter dem Wort Staat verstehen." 18

Nach Werbőczy bedeutete also die Lehre von der Heiligen Krone, dass die Gewalt des Königs von den Gliedern der Heiligen Krone beschränkt werden konnte. Die Glieder der Heiligen Krone waren diejenigen, die ihren Grundbesitzen von der Heiligen Krone erhielten. Wie das von Timon formuliert wird: "Es wurde eine eigentlich öffentliche Gewalt und zwar eine constitutionell beschränkte."19 Die Glieder der Heiligen Krone konnten ihre Rechte an den Landtagen ausüben, also konnte die Gewalt des Königs durch Gesetzen beschränkt werden. Werbőczy formuliert das folgenderweise: "So begannen denn die Könige Gesetze zu schaffen, indem sie das Volk einberiefen und befragten. Und so pflegt es auch zu unseren Zeiten zu geschehen."20

Daraus folgt, dass das Lehnswesen in Ungarn nicht einwurzeln konnte. Das wurde durch die starke öffentlich-rechtliche Konstellation der Heiligen Krone verhindert. Die Besitzverleihung begründete kein Lehnsverhältnis zwischen dem König und dem Begüterten. Diese Verhältnisse wurden nicht durch Kontrakten, sondern durch Gesetze geregelt. ${ }^{21}$

Timon schreibt darüber Folgendes: "Die Schenkungsgüter verwandelten sich nicht in Lehnsgüter. [...] Das königliche Schenkungsrecht wurde nicht als private Machtbefugnis des Königs, sondern als ein Bestandteil der öffentlichen Gewalt aufgefasst, die dem König als dem souveränen Oberhaupt des Staates zusteht. Eben darum ist das Schenkungsgut, ebenso wie das Geschlechtsgut ${ }^{22}$ die Belohnung öffentlicher Verdienste (laurea virtutis), die keine anderen, als die aus dem öffentlichen Verband fließenden öffentlichen Pflichten nach sich zieht und keinerlei lehnähnliches Abhängigkeitsverhältnis zwischen dem König und dem Donatar begründet."23

Péter Váczy sieht den Unterschied zwischen Schenkungsgüter und Benefizium darin, dass dem Donatar das Besitzrecht 'in proprium' erteilt wurde, blieb also das Verfügungsrecht nicht bei dem König, wie im Fall des Lehnswesens, als der Vasall nur das Gebrauchsrecht am Boden erhielt. ${ }^{24}$ Im Einklang mit dieser Festsetzung schreibt auch Bónis: "Die dingliche Seite des Lehnsverhältnisses umfasst die Frage der rechtlichen Natur des Lehnsbesitzes. Die Prinzip, wonach das Benefizium im Eigentum

\footnotetext{
18 Timon 1904, 513-514.

19 Timon 1904, 514.

20 Tripartitum, tit. 3. p. II, $\mathbb{\$}$ 2. (Übers. Timon 1904, 515).

21 Tomcsányi 1940, 274.

22 Ungarisch szállásbirtok: diejenige Boden, die nach der Landnahme den Geschlechten zugeteilt wurden.

${ }^{23}$ Timon 1904, 363.

24 Váczy 1932, 373.
} 
Magdolna Gedeon

Die Rechtslage des Grundbesitzes im Spiegel

der Lehre von der Heiligen Krone
Journal of Agricultural and

Environmental Law

$29 / 2020$

des Lehnsherren steht, und der Vasall leitet sein Verfügungsrecht aus ihrem stärkeren Recht ab, das zu dem Recht der Entlehner ähnlich ist, entsteht schon im fränkischen Recht. Dieses Recht enthält in sich: Besitz, Gebrauch, und Verantwortung für den Bestand." 25

Wenn wir diese Frage ganz vereinfacht und vom praktischen Aspekt prüfen, können wir behaupten: wenn die Adeligen, die das Besitzrecht erhielten, als die Glieder der Heiligen Krone die Gewalt des Königs durch Gesetze beschränken konnten, wenn Gesetze hinsichtlich der Bodenbesitzen gebracht wurden, gehörten diese nicht zu der Person des Königs, sondern $\mathrm{zu}$ der Heiligen Krone. Daraus folgt, dass kein privatrechtliches Lehnsverhältnis, sondern eine öffentlich-rechtliche Beziehung zwischen dem König und den Adeligen bestand.

Aus der Änderungen der Regeln in Bezug auf die Bodenbesitzen können wir darauf folgern, dass die königliche Gewalt am diesen Gebiet beschränkt werden konnte. Die Rechtslage der Grundbesitze wurde durch Artikel festgesetzt. In den Gesetzen Stefans des Heiligen finden wir hinsichtlich der Rechtslage der Geschlechtsgüter und der Schenkungsgüter keinen Unterschied. ${ }^{26}$ Die Erbfolge wurde nicht beschränkt, die Sippengenossen und Seitenverwandten konnten auch die Schenkungsgüter erben, wie die Geschlechtsgüter. Kálmán beschränkte aber das Erbfolgerecht hinsichtlich der Schenkungsgüter: er schloss die Sippengenossen und Seitenverwandten von der Sukzession aus. Die Schenkungsgüter konnte der engere Familienkreis erben, also die männlichen Deszendenten, falls keine vorhanden wären, der Bruder und dessen söhne. Betreff der von Stefan dem Heiligen verliehenen Schenkungsgüter blieben noch die vorigen Regeln aufrecht. ${ }^{27}$ Die Adeligen wollten aber das Erbrecht der Sippengenossenen und Seitenverwandten wiederherstellen. Das erreichten sie durch den Artikel IV. der Goldenen Bulle.28

\footnotetext{
25 Bónis 2003, 47.

26 Stefan I, decr. II, cap. 35. "Wir haben der Wille des Rates zugestimmt, dass jedermann der Herr seines Eigentums seien, genauso auch der Schenkungen des Königs, bis er lebt, ausgenommen die Güter, die zum episcopatum und comitatum gehören. Nach seinem Tod haben seine Söhne ähnliche Rechte" (Márkus 1896-1901, Bd. I, 36). Dieser Artikel beweist auch die Festsetzung von Zlinszky über die geteilte königliche Macht, da dieses Dekret wegen der Wille des Rates erteilt wurde. Siehe noch das sog. Ratsgesetz aus dem Jahr 1298. Art. XXIII.: [...] „wenn der Herr König dies unterliesse, so sei alles, was er ohne den Rat der Vorgenannten in Betreff grosser Schenkungen oder Besetzung von Ämter oder in anderen wichtigen Dingen beschlösse, unverbindlich“ (Übers. Timon 1904,176).

${ }^{27}$ Kálmán, decr. I, cap. 20: "Die Güter, die von Stefan dem Heiligen verliehen wurden, werden von jeden Nachfolgern nach dem Erbfolgerecht vererbt. Die Schenkungen der anderen Könige werden von den Söhnen geerbt, wenn es keinen Sohn gibt, folge der Bruder, nach dessen Tod sollen seine Söhne auch nicht aus der Erbschaft ausgeschlossen werden. Wenn es aber keinen Bruder gibt, gehöre das Besitz dem König” (Márkus 1896-1901, Bd. I, 102-103).

28 Art. IV:1222: "Wenn ein Adelige ohne Sohn stirbt, soll seine Tochter den vierten Teil des Gutes erben, über das andere soll er so verfügen, wie er möchte. Und wenn er ohne Testament stirbt, sollen seine Seitenverwandten erben. Und wenn er überhaupt keine Verwandten hat, wird der König es in Besitz nehmen” (Márkus 1896-1901, Bd. I, 132-133).
} 
Magdolna Gedeon

Die Rechtslage des Grundbesitzes im Spiegel

der Lehre von der Heiligen Krone
Journal of Agricultural and

Environmental Law

$29 / 2020$

Der Artikel IV. der Goldenen Bulle im Jahre 1222 wurde im Jahre 1351 aufgehoben, als der Avitizitätsprinzip eingeführt wurde. ${ }^{29}$ Laut Timon: "Die neue Besitzverfassung, welche unter den Königen aus dem Hause Anjou sich consolidierte, und dann Jahrhunderte lang das Fundament der ständischen Verhältnisse bildete, beruhte auf zwei Grundprincipien: dem Aviticitätsprincip und dem Princip der Heiligen Krone. Das erstere bedeutete die Gebundenheit des Grundbesitzes gegenüber dem Geschlecht (Sippe), letzteres die Gebundenheit gegenüber der die öffentliche Gewalt vorstellenden Heiligen Krone. [...] Die Quelle, das Fundament alles Grundbesitzes ist der Heilige Krone. Das Staatsgebiet ist das Gebiet der Heiligen Krone; ein freies Besitzrecht hat nur derjenige, der seinen Grundbesitz unmittelbar von der Heiligen Krone als Wurzel allen Besitzrecht (radix omnium possessonium) herleitet. Das Geschlecht - d. i. die Descendenz - des Donatars besitzt ein von der Heiligen Krone hergeleitetes radikales Recht an dem Gute, solange ein Glied des Geschlechts vorhanden ist; dann kehrt der Grundbesitz zur Wurzel zurück und unterliegt der neuerlichen königlichen Verleihung." 30

\section{Die Einführung der Lehnsverhältnisse am Gebiet des Bergwesens}

Die Könige waren sich im Klaren über die Bedeutung der Heiligen Krone, und sie wussten sehr gut, dass diese ihre Rechte begrenzt. Die öffentlich-rechtliche Kraft der Heiligen Krone kann durch die Geschichte des Bergregals bewiesen werden.

Aus dem Bergregal stammen die Rechte, die dem König vorbehalten waren. Diese Rechte wurzelten im Interesse und in den eigenartigen Bedürfnissen des Bergbaus. Der König wurde auf diesem Gebiet als 'oberster Bergherr' bezeichnet. ${ }^{31}$ Als oberster Bergherr konnte der König über das Bergwesen regieren. Die vorbehaltenen Bergprodukte gehörten dem König. Dieser hatte also das Recht, diese Schätze auch auf fremden Grundstücken abzubauen. Wenn er die Bergbautätigkeit nicht selbst ausüben wollte, konnte er dieses Recht anderen überlassen und er erhielt dafür einen Teil des Ertrages, meistens einen Zehntel. Das war die urbura oder die Frohn.

Heiner Lück schreibt über das Bergregalrecht in Deutschland, dass die Bergunternehmer, da ihre Tätigkeit spezielle Kenntnisse erforderten, viele Privilegien erhielten. Die Herrscher erteilten die Privilegien den Unternehmern, damit die Bergleute ihren Wohnsitz verlassen und unter dem Schutz des Herrschers die Bergwerke betreiben. Die wichtigsten Punkte der Privilegien waren, dass die hospes ihre eigenen, hauptsächlich aus Deutschland mitgebrachten Rechte anwenden dürfen. ${ }^{32}$

\footnotetext{
${ }^{29}$ Ludvig I, praef. der decrete von 1351 \11: Die Regeln der Goldenen Bulle von 1222 werden befestigt, ausgenommen der Artikel IV, wonach die Adeligen über seinen Güter frei verfügen können. "Sie haben kein Recht das zu tun, sondern ihre Besitze verfallen nach Recht und Gesetz bedingungslos, ohne irgendwelchen Widerspruch, den nächsten Verwandten und Sippengenossen” (Márkus 1896-1901, Bd. I, 170-171).

30 Timon 1904, 552-556.

31 Wenzel 1866, 73.

32 Lück 2008, 529.
} 
Magdolna Gedeon

Die Rechtslage des Grundbesitzes im Spiegel

der Lehre von der Heiligen Krone
Journal of Agricultural and

Environmental Law

$29 / 2020$

Auf diese Weise wurde z. B. auch die Bergstadt Schemnitz gegründet. König Béla IV. rief nämlich deutsche Siedler ins Land, die nach 1244 die von den Mongolen zerstörte Stadt wieder aufbauten. Sie bekamen neue Privilegien und fassten ihre mitgenommenen Rechte und die am Ort entstandenen Rechtsgewohnheiten zusammen. Der König bestätigte die auf diese Weise geschaffenen Statuten der Stadt. In diesem Privilegium finden wir schon eine Hinweis auf die Heilige Krone: "[...] vnd gebnn In vnd verleihnn, Recht vnd freyheitt als hernoch geschribnn set, Die der heilignn Krön vnd ihrem nwtz wol fuegnn vnd frwmmen." 33 Der König vindizierte also die Einnahmen aus der Bergwerke nicht für sich, sondern für die Heilige Krone. Nach Timon "sah das ungarische Volk den Staat in dieser Zeit, die im Interesse der Gesamtheit organisierte Gesellschaft, als organisches Ganze in der Heiligen Krone verkörpert."34

Die Beschränkung der Bergregalrechte des Königs finden wir auch im Artikel I:1514: "Die Einnahmen der königlichen Heiligen Krone wurden bis jetzt für verschiedenen Menschen festgebunden und einstweilen entfremdet. [...] wir entschieden vor allem, dass alle Einkünfte des Königs [...] die Bergwerke und Salzkammern, Gold- und Silberbergwerke, und die königlichen Städte zurückgegeben werden sollen." 35

Die ungarischen Könige hatten anhand des Regalrechts auch die Befugnis erhalten, die Bergbautätigkeit zu verwalten. Wegen der Erhöhung der Einnahmen des Staatshaushalts erschienen die Zentralisationsbestrebungen auf dem Gebiet des Bergwesens schon im 16. Jahrhundert. Im 16. Jahrhundert stiegen nämlich die Kosten der Bergwerke, da es sehr aufwendig war, Erz aus den tieferliegenden Schichten abzubauen. Deshalb wollten die Unternehmer immer mehr Erträge behalten und der Hof versuchte seine Aufsicht über den Bergbau auszuweiten. In Westeuropa funktionierte das Regalrecht nach den Prinzipien des Lehnswesens: Der Herrscher regierte das Bergwesen als sein Eigentum, er konnte über die Einnahmen aus der Montanindustrie selbst verfügen. Die Herrscher des Hauses Habsburg, die auch ungarische Könige, sowie gleichzeitig oberste Bergherren waren, wollten diese Auffassung auch in Ungarn einführen. Deswegen wollten die Herrscher das Bergwesen ohne Stände und mit Verordnungen verwalten.

Der erste Schritt war auf diesem Weg die Einführung der Maximilianische Bergordnung, die nach mehreren Umarbeitungen im Jahre 1560 fertiggestellt wurde und sie wurde nach langem Verhandeln von Kaiser Maximilian II. am 10. Februar 1565 verkündet. ${ }^{36}$ Diese Ordnung konnte aber nicht in Kraft treten, da die Bergstädte nicht zu einer Einigung bereit waren, obwohl schon 1564 in einer Verordnung des Kaisers bestimmt wurde, dass die neue Bergordnung überall in den Bergstädten verkündet und in 32 Exemplaren ausgeteilt werden solle. ${ }^{37}$ Die Maximilianische Bergordnung konnte schließlich am 16. Februar 1573 in sieben niederungarischen Bergstädten verkündet werden, nachdem die aus den alten Bergrechten der Bergstädte zusammengestellten

\footnotetext{
${ }^{33}$ Fuchs 2009, 25.

34 Timon 1904, 511.

35 Márkus 1896-1901, Bd. I, 707.

${ }^{36}$ Kundmachungspatent, 10. Februar 1565. (Schmidt 1832-1839, Bd. 2, 1).

${ }^{37}$ Kaiserliche Reskript, 10. September 1564. (Schmidt 1832-1839, Bd. 1, 412).
} 
Erläuterungen als Anhang zu der neuen Bergordnung hinzugefügt worden waren. ${ }^{38}$ Eine Passage der neuen Bergordnung besagt, dass diese nur in dem Fall angewandt werden solle, wenn man in den Erläuterungen keine passende Regel finden würde. ${ }^{39}$ Also kam die Maximilianische Bergordnung nur subsidiär zur Geltung.

Die Maximilianische Bergordnung enthält praktische, dem damaligen Bergbau zeitgemäße, geeignete Vorschriften. Warum verteidigten die Bergstädte ihre Rechte gegen die neue Bergordnung so hartnäckig? Gleich auf der ersten Seite der Maximilianischen Bergordnung findet sich die Information, dass alle Bergwerke und künftigen Stollen, samt den zu ihrem erfolgreichen Abbau aufzubrauchenden Wäldern und Wasserleitungen dem Kammergut gehören, und dass es den weltlichen und kirchlichen Herren und Adeligen und den Städten verboten ist, mit dem Abbau zu beginnen, ohne eine besondere königliche Erlaubnis ein Bergwerk aufzuschlagen, abzubauen und dort zu arbeiten, und von den Bergmännern und Amtsleuten der Hofkammer Urbar oder Fron zu fordern. ${ }^{40}$

Mit Hilfe dieses Paragraphen sollte eine vorher in Ungarn nicht gebräuchliche Regel eingeführt werden, nach der - wie das von Delius erklärt wird - 'das Bergwerksregale dem Landesherrn unmittelbar vorbehalten wird.'41 Gábor Bóday, Übersetzer des Werkes von Delius fügt aber dieser Bemerkung zu: "Das ungarische Verfassungsrecht kannte jedoch keine »unmittelbar vorbehaltenen" Regalrechte und die Bergwerkshoheit konnte auch vom König ausschließlich im Rahmen der ungarischen Gesetze durch die offiziellen Behörden, unter der Aufsicht des Landestages, ausgeübt werden." 42

Die Maximilianische Bergordnung wurde im Jahre 1723 durch den CVIII. Artikel als lex privata der Berggerichte als Gesetz akzeptiert: "Die Bergerichte bleiben, nach deren mehr als vor einhundert Jahren festgelegten Privatgesetzen, in ihrer derzeitigen Form erhalten."43 Mit Hilfe des Artikels von 1723 konnte die Bergordnung bis 1854, bis zum Inkrafttreten des österreichischen Berggesetzes, gültig bleiben.

Aufgrund der Maximilianischen Bergordnung konnte der Habsburger Hof auch eine von den Ständen völlig separierte, zentralisierte Bürokratie in der Bergverwaltung ausbauen. So konnten die Habsburger Herrscher erreichen, dass sie über die Einkünfte aus der Bautätigkeit allein, ohne den Ständen verfügen konnten. Der König, als der oberste Bergherr verwaltete das Bergwesen mit Verordnungen. Also gehörten die Bergschätze nicht mehr der Heiligen Krone, sondern der Person des Königs, zwischen dem Bergunternehmer und dem Herrscher entstand nicht mehr ein öffentlich-

38 Kundmachungspatent, 16. Februar 1573. (Schmidt 1832-1839, Bd. 2, 224). Hier kann man auch den Text der Bergordnung lesen. Eine weitere Ausgabe mit den Erläuterungen und mit den Reskripten von Königin Maria Theresia: Maximilian II, 1805.

${ }^{39}$ Maximilian II, 1805, 28, I. $\int 5$.

${ }^{40}$ Maximilian II, 1805, 28. I. \$1.

${ }^{41}$ Delius 1806, Bd. 2, 426. Delius setzt hier auch fest, dass ein Lehnsverhältnis zwischen dem König und dem Bergunternehmer entsteht: "So stehet es ihm auch frey (dem Landesherr), den Bergbau in seinen Ländern entweder selbst unmittelbar zu betreiben, oder denselben seinen Unterthanen unter Vorbehaltung gewisser Rechte und Abgaben in die Lehen zu reichen.”

42 Delius 1972, 455, Anm. 129.

43 Márkus 1896-1901, Bd. 4, 646, Art. CVIII:1723: „Judicia montanistica secundum privatas eorundem leges, ultra seculum stabilitas: in suo esse manebunt.” 
Magdolna Gedeon

Die Rechtslage des Grundbesitzes im Spiegel

der Lehre von der Heiligen Krone
Journal of Agricultural and

Environmental Law

$29 / 2020$

rechtliches, sondern ein Lehnsverhältnis. Das kann auch durch eine Verordnung bewiesen werden.

Die von den Privatpersonen angetriebenen Gewerkschaften spielten eine große Rolle in der Erhöhung der Einnahmen des Königs, da er aus der nach den Metallen eingezahlte Fron und aus der Zwangseinlösung bedeutenden Gewinn erhielt. Aus der Regulierung der Gewerkschaften wird geklärt, dass der Habsburger Hof - wegen der Sicherung der Einnahmen - die Gewerke mit verschiedenen Mittel veranlassen wollte. Die Staatsorgane griffen aber nur zur Sicherung der Rückerstattung der staatlichen Finanzierung in den Betrieb der Gewerkschaften ein.

Die Gewerken waren solche Personen, die nur finanziell im Betrieb der Gewerkschaft teilnahmen. Ihre Teile wurden durch die Kuxe verkörpert. ${ }^{44}$ Über die Rechtslage der Kuxe finden wir mehreren Verordnungen in den Quellen. Neben den Verordnungen, die den Kauf der Bergteile regelten, wurden von der Hofkammer auch solche erteilt, die die Beerbung der Kuxe verordneten. Nach einer Verordnung aus dem Jahr 177945 fielen die Kuxe nicht unter das ius condivisionis, ${ }^{46}$ die zivilrechtlichen Regeln bezogen sich auf sie auch nicht in den Ländern, wo die Maximilianische Bergordnung als Gesetz in Kraft tritt. Auf diesem Grund bezogen sich auf sie die Regeln der Avitizität auch nicht. Daraus folgte, dass die Gewerken über ihre Kuxe frei verfügen konnten. Die Bergteile konnten frei verkauft, verschenkt, verpfändet werden. Die Gewerken durften auch die Kuxe frei testieren. Sie hatten nur im Fall Vorkaufsrecht auf die Kuxe, wenn ihr Eigentümer in Retardat ${ }^{47}$ geriet. Wenn ein Gewerke mit ihren Teilen aufhörte, und er keine Fristung bei der Berggericht erhielt, konnte dieser Teil wieder gemutet werden.

Die Verordnung setzt also fest, dass die Landgesetze auf die Bergteile keine Wirkung hatten, so wurden die Bergteile des Gewerkes von den Beschränkungen völlig befreit. In diesem Fall wurde die Wirkung der Gesetze durch eine Verordnung aufgehoben. Die Diskrepanz wurde in der Rechtsquellenordnung dadurch aufgelöst, dass "die Bergverwandte als freie, landesfürstliche Lehnsleute zu betrachten sind, auf welche die Landes- und Zivilgesetze keinen Einfluss haben." 48 Auf diesem Grund kann ausschließlich der König im privatrechtlichen Lehnsverhältnis Regeln erteilen, die sich auf die Gewerken beziehen. Der König hatte also das Recht, diese Verhältnisse durch Verordnungen zu verwalten.

\footnotetext{
44 "Kux ist der bestimmte ideelle Teil, Anteil an einer gewerkschaftlichen Zeche oder Grube, welche früher aus 128, jetzt aus 100 Kuxen oder Teilen nach dem Decimalstyle besteht und substantiell nur ein Ganzes bildet”' (Erklärendes Wörterbuch 1869, 91).

${ }^{45}$ Hofkammerdekret, 14. Mai 1779. (Schmidt 1832-1839, Bd. 14, 292).

${ }^{46}$ Ius condivisionis: die nächsten Geschlechtsgenossen sollten bei der Erbteilung ihr Vermögen einrechnen. Der Geschlechtsgenossen konnten über ihren Sondervermögen bis zur Erbteilung frei verfügen (vgl. Homoki 2005, 55). Nach der zitierten Verordnung sollten die Bergteile nicht eingerechnet werden.

${ }^{47}$ Retardat: der Gewerke zahlt die die Kuxe belasteten Kosten nicht aus.

48 Hofkammerdekret, 14. Mai 1779. (Schmidt 1832-1839, Bd. 14, 292).
} 
Magdolna Gedeon

Die Rechtslage des Grundbesitzes im Spiegel

der Lehre von der Heiligen Krone
Journal of Agricultural and

Environmental Law

$29 / 2020$

Josef der II. wollte seine Macht auch mit Hilfe der Verordnungen im Bergwesen stärken, die Hofkammer verbreitete ihre Macht vollständig am Gebiet des Bergbaus. Diese Methode wollte aber von den Ständen nach dem Tod von Josef II. gerade durch die Bergrechtskodifikation geändert werden, da der Landtag hoffte, auf dieser Weise seinen Einfluss im Bergbau zu verstärken.

Nach Emma Bartoniek ${ }^{49}$ hatte die Lehre von der Heiligen Krone in der Neuzeit kleinere Bedeutung, als früher. Die Heilige Krone bleibt aber das Symbol des ungarischen Staates, und war die Quelle aller Grundbesitze. In diesem Sinn wurde am meisten erwähnt. ${ }^{50}$

Nach dem Tod von Josef dem II. wollten die Stände durch die Gesetzgebung die Vollmacht des Herrschers beschränken. ${ }^{51}$ Die Lehre von der Heiligen Krone spiegelt sich auch im Artikel XII:1790. ${ }^{52}$ Der Kodifikationsversuch begann schon am Landtag 1790/1791, als der Artikel LXVII. auf dem Grund des Artikels XXII. ${ }^{53}$ verordnete, dass eine Deputatio Montanistica ein Berggesetz verfassen soll. Obwohl die Kommission einen Entwurf mit 53 Artikeln zusammenstellte, wurde das nicht als Gesetz empfangen. Der Artikel IX. im Jahre 182754 ernannte einen neuen Kommission, der die bisherigen Regeln systematisch ordnete und einen Entwurf mit 189 Paragraph vorbereitete. Das wurde aber sogar nicht vom Landtag geprüft. ${ }^{55}$

Im Jahre 1844 wurde der erste wahre ungarische Berggesetzentwurf dem Landtag vorgelegt. Die Hofkammer schickte aber einen Ausschuss wegen der Untersuchung des geplanten Gesetzes aus. Aus der Meldung dieses Ausschusses kommt hervor, dass gerade die Rechte des Königs durch das neue Berggesetz beschränkt worden wären, und in der Regierung des Bergbaus hätte der Landtag mehrere Befugnisse erhalten. Dieser Gesetzesentwurf hätte also die Vollmacht der

49 Emma Bartoniek (1894-1957), Historikerin und Bibliographin. Sie wollte nicht Stellung nehmen, publizierte ihre eigene Meinung über die Lehre von der Heiligen Krone (Bódi 2015,7).

${ }^{50}$ Bartoniek 1987, 168.

${ }^{51}$ Die Bedeutung der Heiligen Krone wird aber dadurch gezeigt, dass Josef der II. die Krone nach Wien liefern ließ, die nach seinem Tod nach Budapest geliefert wurde. Dieser Weg der Heiligen Krone wurde als der Siegeszug der Unabhängigkeit der Stände, die ungarische Selbständigkeit und Freiheit betrachtet (Kardos 1992, 37).

52 „Die Macht der Gesetzgebung, der Aufhebung und Erklärung der Gesetze betreffen in Ungarn und in den angeschlossenen Teilen, ohne der Beschwer des Artikels VIII:1741, den gesetzmäßigen gekrönten König und zu dem Landtag einberufenen Stände gemeinsam, und das kann außer ihnen von niemand geübt werden" (Márkus 1896-1901, Bd 3, 160-161). Dieser Artikel wurde im Weiteren als die gesetzgeberische Grundformel der Lehre von der Heiligen Krone betrachtet (Kardos 1992, 43, 37).

${ }^{53}$ Die Bestrebung der ungarischen Ständen, den Einfluss in Bergwesen zu erhalten, spiegelt sich auch in diesem Artikel, der in sich hält, dass der König den Ständen versprach, das Bergwesen unter die ungarischen Kammer zu stellen, die Berggesetze mit dem Landtag zu verbessern. Am Ende des Artikels steht aber, dass „der König die wirtschaftliche Regelung des Bergwesens, als $\mathrm{zu}$ den königlichen Befugnissen gehörende Sache, im Bereich seiner Entscheidung aufhält" (Márkus 1896-1901, Bd. 3, 202-203; 166-167). Das bedeutete, dass der König, als oberster Bergherr, über die Einnahmen weiterhin selbst verfügen wollte und die wirtschaftlichen Umstände mit den Verordnungen regeln konnte.

54 Márkus 1896-1901, Bd. 3, 442-443.

55 Vgl. Balkay 1904, 464; Wenzel 1866, 60. 
Magdolna Gedeon

Die Rechtslage des Grundbesitzes im Spiegel

der Lehre von der Heiligen Krone
Journal of Agricultural and

Environmental Law

$29 / 2020$

fremden Hofkammeroffiziere beseitigt, und das hätte bedeuten, dass die verantwortungslose Wirtschaftsführung der Hofkammer beendet worden wäre, und die nationalen, verfassungsrechtlichen Aspekte in Betracht nehmenden Verhältnisse sich durchgebrochen hätten, die Einnahme aus dem Bergbau nicht allein den König bereichert hätten, sondern das ganze Land. Dieser Artikel hätte also das Bergwesen wieder unter die Heilige Krone geordnet.

Wegen dieser Meldung des Ausschusses wurde der Entwurf nicht sanktioniert. Wie Gusztáv Wenzel erklärte: “dieser Entwurf regelte nicht mehr, was das Bergregal ist, sondern was sind die Objekte der königlichen Verleihungen und Bergerlaubnissen. Damit wäre diese Frage vom Gebiet des zivilrechtlichen abstrakten Begriffes an das Gebiet des von der Bergbaufreiheit geordneten Rechtslebens durchgeführt worden." 56

Die historischen Verhältnisse ermöglichten, im Jahre 1854 das allgemeine österreichische Berggesetz in Ungarn eizuführen. Das von der ungarischen Entwicklung fremde Gesetz befestigte die Vollmacht der Habsburger im Bergbau. Die feudalen Bindungen wurden aber beseitigt, und die industrielle Entwicklung einen Schwung bekommen konnte. ${ }^{57}$ In drittem Paragraph können wir lesen den Begriff des Bergregals, wonach die Bergschätze der Person des Königs gehörten. ${ }^{58}$

Die Geschichte des Bergregals unterstützt eher die Auffassung von Ákos Timon.

Wie das nämlich oben geschildert wird, kämpften die Habsburger Herrscher bewusst dafür, dass die aus dem Bergwesen einfließenden Einkünfte nicht der Heiligen Krone sondern ihnen gehören. Das hätten sie hinsichtlich der Grundbesitze nicht erreichen können, da die Adeligen und die Städte gerade durch die Schenkungsgüter die Glieder der Heiligen Krone waren. Auf diesem Grund kann festgesetzt werden, dass die Grundbesitze nicht dem König, sondern der Heiligen Krone gehörten, zwischen den Begüterten und dem König keine privatrechtliche, sondern eine öffentlich-rechtliche Beziehung entstand, die Rechte des Königs bezüglich der Grundbesitzes durch Gesetze beschränkt werden konnten.

\section{Der Artikel P des Grundgesetzes und die Lehre von der Heiligen Krone}

Der Artikel P des Grundgesetzes entspricht der Lehre von der Heiligen Krone: "Die natürlichen Kraftquellen, insbesondere Ackerboden, Wald und Trinkwasservorräte, sowie die biologische Artenvielfalt, insbesondere einheimische Pflanzen- und Tierarten, und die kulturellen Werte bilden das gemeinsame Erbe der Nation, dessen Schutz und Bewahrung für die zukünftigen Generationen die Pflicht des Staates und aller Menschen darstellt."

\footnotetext{
${ }_{56}$ Balkay 1904, 464-466; Wenzel 1866, 76.

${ }^{57}$ Izsó 2004, 16.

58 "Unter Bergregale wird jenes landesfürstliche Hoheitsrecht verstanden, gemäß welchem gewisse, auf ihren natürlichen Lagerstätten vorkommende Mineralien der ausschließlichen Verfügung des allerhöchsten Landesfürsten vorbehalten sind”' (Grenzenstein 1855, 79).
} 
Magdolna Gedeon

Die Rechtslage des Grundbesitzes im Spiegel der Lehre von der Heiligen Krone
Journal of Agricultural and

Environmental Law

$29 / 2020$

Die Lehre von der Heiligen Krone ist nämlich eine besondere Souveränitätstheorie, die auch die Einheit der Nation ausdrückt. Die Glieder der Heiligen Krone sind heute die Glieder der Nation. ${ }^{59}$ Die natürlichen Kraftquellen, auch die Ackerboden, sollen für die nächsten Generationen versichert werden. Also ist der Umweltschutz die Aufgabe aller Menschen, aller natürlichen und Rechtspersonen. ${ }^{60}$

59 Timon formuliert das folgenderweise: "Dereinst jemand, der sein Besitzrecht von der Heiligen Krone ableitete, war der Mitglied der Heiligen Krone (membrum Sacrae Regni Coronae), und er nahm auf diesem Grund in der Übung der dem Heiligen Krone gebührenden Hoheitsrechte teil. Seit der Annahme der bürgerlichen Rechtsgleichheit bilden die ganze Nation, jeden Einwohner des Gebiets der Heiligen Krone samt mit der Heiligen Krone gekrönten König das öffentlichrechtliche Ganze, die lebendige Organisation, die vom unseren alten öffentlichen Recht als der Körper der Heiligen Krone (totum corpus Sacrae Regni Coronae) gezeichnet wurde, die in unseren Tagen Staat genannt wird" (Timon 1920, 14).

${ }^{60}$ Vgl. Gáva, Smuk \& Téglási 2017, 17. 


\section{Bibliographie}

1. Balkay B (1904) Bányatörvényünk reformálásának első kísérletei, Bányászati és Kohászati Lapok, Bányászat, 37(39), pp., 462-469.

2. Bartoniek E (1987) A magyar királykoronááások története, Magyar Történelmi Társulat, Budapest.

3. Bódi S (2015) A Szent Korona-eszme különbségei Echart Ferenc, Timon Ákos és Bartoniek Emma felfogásában, Polgári Szemle, 11(1-3), pp. 327-341.

4. Bónis Gy (2003) Hübériség és rendiség a középkori magyar jogban, Osiris, Budapest.

5. Delius C T (1806) Anleitung zu der Bergbaukunst nach ibrer Theorie und Ausübung, Bd. 2, Hof- und Staats-Druckerey, Wien.

6. Delius Kristóf Traugott (1972) Értekezés a bányászati kamerális tudomány alapelveiről (ford. Bóday Gábor), in: Bevezetés a bányatan elméletébe és gyakorlásába valamint a bányakincstári tudományok alaptételeinek ismertetésébe, OMBKE, Bányászati Szakosztály, Budapest, pp. 445-479.

7. Érszegi G (Hrsg.) (1987) Árpád kori legendák és intelmek, Szépirodalmi Könyvkiadó, Budapest.

8. Grenzenstein G (Hrsg.) (1855) Das allgemeine österreichische Berggesetz, vom 23. Mai 1854, und die Verordnungen über die Bergwerksabgaben vom 4. October 1854, Friedrich Manz, Wien.

9. Echart F (1931) Jog- és alkotmánytörténet, in: Hóman Bálint (Hrsg.) A magyar történetírás új útjai, Magyar Szemle Társaság, Budapest, pp. 269-323.

10. Echart F (1941) A Szentkorona-eszme története, MTA, Budapest.

11. Erklärendes Wörterbuch (1869) Erklärendes Wörterbuch der im Bergbau, in der Hüttenkunde und in Salinenwerken vorkommenden technischen Kunstausdrücke und Fremdwörter, Falkenberg, Burgsteinfurt, 1869.

12. Schmidt F A (1832-1839) Chronologisch Systematische Sammlung der Berggesetze der Österreichischen Monarchie. II. Abteilung: Chronologisch Systematische Sammlung der Berggesetze der Königreiche: Ungarn, Kroatien, Dalmatien, Slavonien und des Grossfürstenthums Siebenbürgen, Band I-XXV, J. P. Sollinger, Wien.

13. Fuchs P (Übers.) (2009) Das Rechtsbuch von Schemnitz - Selmecbánya város középkori jogkönyve, Montan-Press, Budapest.

14. Gáva K, Smuk P \& Téglási A (2017) Az. Alaptörvény értékei, Dialog Campus Kiadó, Budapest.

15. Hajnik I (1867) Magyarország az. Arpád-királyoktól az ösiségnek megállapitásáig és a bübéri Európa, Heckenast Gusztáv, Pest.

16. Lück H (2005) Bergrecht, Bergregal, in: Cordes A, Haferkamp H-P, Lück H, Werkmüller D, Schmidt-Wiegand R (Hrsg.), Handwörterbuch zur deutschen Rechtsgeschichte, Band I. Erich Schmidt Verlag, Berlin, pp., 527-533.

17. Lück H (2015) Die Entwicklung des deutschen Bergrechts und der Bergbaudirektion bis zum Allgemeinen (preußischen) Berggesetz 1865, in: Wolfhard W (Hrsg.) Geschichte des deutschen Bergbaus, Bd. 2. Salze, Erden und Kohlen. Der Aufbruch in die Moderne im 18. und frühen 19. Jahrhundert. Münster, Aschendorff, 2015, pp., 111-216.

18. Homoki Nagy M (2005) A magyar magánjog történetének vázlata 1848-ig, JATE Press, Szeged. 
Magdolna Gedeon

Die Rechtslage des Grundbesitzes im Spiegel der Lehre von der Heiligen Krone
Journal of Agricultural and

Environmental Law

$29 / 2020$

19. Izsó (2004) The introduction and influence of the Austrian Common Mining Law in Hungary, in: The 150th anniversary of issue of the Common Mining Law (Konference, Hradec nad Moravici, Czech Republic, 9. 2004.) Montanex, Ostrava, pp. 15-17.

20. Kardos J (1992) A Szent Korona és a Szent Korona-eszme története, IKVA, Budapest.

21. Márkus D (Hrsg.) (1896-1901) Corpus Juris Hungarici, I-VIII, Franklin, Budapest.

22. Maximilian II. (1805) Neue Berg-Ordnung des Königreichs Ungarn, und solcher Cron einverleibten Gold, Silber, Kupfer, und anderer Metall- Bergwerken, Sammt denen Erläuterungen zweyer Alten Berg-Ordnungen Der sieben königl. Freyen Berg-Städte 1. Chemnitz u. Königsberg 2. Schemnitz, Neusobl, Bugganz, Dülln und Libeten, Aus der k. auch k. k. Hof- und Staats Druckerey, Wien.

23. Timon Á (1904) Ungarische Verfassungs- und Rechtsgeschichte mit Berug auf die Rechtsentwicklung der westlichen Staaten, Puttkammer und Mühlbrecht, Berlin.

24. Timon Á (1907) A Szent Korona és a koronázás közjogi jelentösége, Rákosi Jenő Budapesti Hírlap Újságvállalata, Budapest.

25. Timon Á (1920) A Szent-Korona elmélete és a koronázás, Stephaneum Nyomda Rt, Budapest.

26. Tomcsányi M (1940) Magyarország közjoga, Királyi Magyar Egyetemi Nyomda, Budapest.

27. Törő L D (2016) Az „Echart-vita“, Echart Ferenc 1931-es programtanulmányának kortárs visszhangja, Aetas 31(4), pp., 57-77.

28. Tóth Z J (2008) Magyar közjogi hagyományok és nemzeti öntudat a 19. század végétöl napjainkig, Adalékok a Szent Korona-esżme történetéhez, Szent István Társulat, Budapest.

29. Váczy P (1932) A húbériség szerepe Szent István királyságában, Saázadok, 66(1-3), pp., 369-392.

30. Wenzel G (1866) A magyar és erdélyi bányajog rendszere, Kir. Magyar Egyetemi Nyomda, Buda.

31. Zétényi Zs (1997) A S žentkorona-esżme mai értelme, Püski Kiadó, Budapest.

32. Zlinszky J (2013) A Szentkorona-eszme és története, in: Koltay András (Hrsg.): A XII táblától a 12 ponton át a magánjog új törvénykönyvéig, Szent István Kiadó, Budapest, pp., 227-245. 